\title{
Turbulent particle transport as a function of toroidal rotation in DIII-D H-mode plasmas
}

X. Wang

Saskia Mordijck

William \& Mary, smordijck@wm.edu

L. Zeng

L. Schmitz

et al.

Follow this and additional works at: https://scholarworks.wm.edu/aspubs

Part of the Physics Commons

\section{Recommended Citation}

Wang, X.; Mordijck, Saskia; Zeng, L.; Schmitz, L.; and et al., Turbulent particle transport as a function of toroidal rotation in DIII-D H-mode plasmas (2016). Plasma Physics and Controlled Fusion, 58(4). https://www.doi.org/10.1088/0741-3335/58/4/045026

This Article is brought to you for free and open access by the Arts and Sciences at W\&M ScholarWorks. It has been accepted for inclusion in Arts \& Sciences Articles by an authorized administrator of W\&M ScholarWorks. For more information, please contact scholarworks@wm.edu. 
PAPER

\section{Turbulent particle transport as a function of toroidal rotation in DIII-D H-mode plasmas}

To cite this article: X Wang et al 2016 Plasma Phys. Control. Fusion 58045026

View the article online for updates and enhancements.

\section{Related content}

\author{
Particle transport in low-collisionality $\mathrm{H}$ - \\ mode plasmas on DIII-D \\ S. Mordijck, X. Wang, E.J. Doyle et al. \\ Understanding ECH density pump-out in \\ DIII-D H-mode plasmas \\ X. Wang, S. Mordijck, E.J. Doyle et al. \\ Role of turbulence regime on determining \\ the local density gradient \\ X. Wang, S. Mordijck, E.J. Doyle et al.
}

\section{Recent citations}

- $\frac{\text { Isotope and fast ions turbulence }}{\text { suppression effects: Consequences for }}$
$\frac{\text { high- ITER plasmas }}{\text { J. Garcia et al }}$
- $\frac{\text { Role of turbulence regime on determining }}{\text { the local density gradient }}$
X. Wang et al
- Understanding ECH density pump-out in
$\frac{\text { DIII-D H-mode plasmas }}{\text { X. Wang et al }}$

\section{IOP ebooks}

Bringing you innovative digital publishing with leading voices

to create your essential collection of books in STEM research.

Start exploring the collection - download the first chapter of every title for free. 


\title{
Turbulent particle transport as a function of toroidal rotation in DIII-D H-mode plasmas
}

\author{
X Wang ${ }^{1}$, S Mordijck ${ }^{2}$, L Zeng ${ }^{3}$, L Schmitz ${ }^{3}$, T L Rhodes ${ }^{3}$, E J Doyle ${ }^{3}$, \\ R Groebner ${ }^{4}$, O Meneghini ${ }^{4}$, G M Staebler ${ }^{4}$ and S P Smith ${ }^{4}$ \\ 1 Department of Physics, College of William \& Mary, Williamsburg, VA 23187, USA \\ 2 Department of Computer Science, College of William \& Mary, Williamsburg, VA 23187, USA \\ 3 Department of Physics and Astronomy, University of California, Los Angeles, CA 90095, USA \\ ${ }^{4}$ General Atomics, PO BOX 85608, San Diego, CA, USA \\ E-mail: xwang09@email.wm.edu
}

Received 16 October 2015, revised 4 January 2016

Accepted for publication 18 January 2016

Published 1 March 2016

\begin{abstract}
In this paper we show how changes in toroidal rotation, by controlling the injected torque, affect particle transport and confinement. The toroidal rotation is altered using the co- and counter neutral beam injection (NBI) in low collisionality H-mode plasmas on DIII-D (Luxon 2002 Nucl. Fusion 42 614) with dominant electron cyclotron heating (ECH). We find that there is no correlation between the toroidal rotation shear and the inverse density gradient, which is observed on AUG when $T_{\mathrm{e}} / T_{\mathrm{i}}$ is varied using ECH (Angioni et al 2011 Phys. Rev. Lett. 107 215003). In DIII-D, we find that in a discharge with balanced torque injection, the $E \times B$ shear is smaller than the linear gyrokinetic growth rate for small $k_{\theta} \rho_{s}$ for $\rho=0.6-0.85$. This results in lower particle confinement. In the co- and counter- injected discharges the $E \times B$ shear is larger or close to the linear growth rate at the plasma edge and both configurations have higher particle confinement. In order to measure particle transport, we use a small periodic perturbative gas puff. This gas puff perturbs the density profiles and allows us to extract the perturbed diffusion and inward pinch coefficients. We observe a strong increase in the inward particle pinch in the counter-torque injected plasma. Finally, the calculated quasilinear particle flux, nor the linear growth rates using TGLF (Staebler et al 2005 Phys. Plasmas 12 102508) agree with experimental observations.
\end{abstract}

Keywords: particle transport, ExB shear, turbulence

(Some figures may appear in colour only in the online journal)

\section{Introduction}

In this paper we study the effects of rotation upon particle transport. Toroidal rotation not only plays a crucial part in stabilizing MHD instabilities, reducing error field penetration but also in determining global confinement in tokamaks [4-8]. The toroidal rotation can contribute to the suppression of turbulent transport through the $E \times B$ shear and thus affects confinement and transport. However, the toroidal rotation and rotational shear can also introduce instabilities. With the current uncertainty in predicting the density profile in tokamaks and its role in determining the fusion gain, it is critical to investigate how different plasma parameters affect particle transport and confinement $[9,10]$.
In large tokamaks the toroidal rotation is driven by a strong injected torque from the neutral beam injection(NBI). On the other hand, future burning plasma devices, such as ITER, will not be able to rely on external injected torque to control the toroidal rotation. It is therefore important to investigate how changes in toroidal rotation, due to changes in injected torque, will affect particle confinement and transport. Previous research on AUG has shown that there is a correlation between the rotational shear and the local density gradient $[2,11]$. The changes in rotational shear and the local density gradient are driven by changes in the characteristics of the dominant turbulent mode in these plasmas on AUG, and not the result of a change in injected torque. On DIII-D, to exclude the effects of a change in the dominant linear mode, we kept the input power 
and fueling levels the same in a set of dedicated discharges. In these discharges, we only change the injected torque, by using a mix of co- and counter beam injection.

Previous research with regards to particle transport concentrates on investigating the role of turbulence and collisionality $[2,11-16]$. In a multi-machine comparison, density peaking increases inversely with collisionality. This trend has been confirmed in a 3-point self-similar scan on JET, where collisionality is varied by a factor 5 and the peaking of the density increases when the collisionality is lowered in $\mathrm{H}$-mode [17]. In DIII-D, where collisionality is only varied by a factor 2 , no change in peaking is observed [15]. Gyrokinetic simulations of these dimensionless collisionality scaling experiments in DIII-D agree that no increase in particle transport should be observed. To test the role of turbulence upon particle transport, experiments on AUG and DIII-D vary the $T_{\mathrm{e}} / T_{\mathrm{i}}$ ratio in order to alter the turbulence drive [11, 13, 18, 19]. The change in $T_{\mathrm{e}} / T_{\mathrm{i}}$ results in a change of the linear stability close to mid-radius, such that the frequency of the dominant mode is altered. The dominant turbulence mode without electron cyclotron heating $(\mathrm{ECH})$ is originally at mid-radius in the ion temperature gradient (ITG) regime. By adding $\mathrm{ECH}$, which pre-dominantly heats the electrons at low collisionality, the electron temperature gradient increases. This reduces the mode frequency and increases the density gradient until the frequency switches sign and the dominant mode changes to the trapped electron mode (TEM), at which point the density gradient decreases strongly [2, 11]. Simultaneously, AUG also observes changes in the local rotation gradient. A correlation between the toroidal rotational shear, $u^{\prime} \equiv \frac{R}{v_{\mathrm{i}, \mathrm{th}}} \frac{\partial v_{\text {tor }}}{\partial R}$, where $R$ is the major radius, $v_{\text {tor }}$ is the toroidal rotation and $v_{\mathrm{i}, \text { th }}$ is the thermal ion velocity and the inverse density gradient $R / L_{n}=-\frac{R}{n} \frac{\partial n}{\partial R}$ is observed on AUG [2]. In a set of discharges on DIII-D where the $T_{\mathrm{e}} / T_{\mathrm{i}}$ ratio is varied by exchanging NBI heating for $\mathrm{ECH}$, we observe a very weak correlation between $R / L_{n}$ and $u^{\prime}$, see figure 1 . The correlation is much weaker than what AUG observes, which might be due to slight difference in input torque in this larger database of discharges. This motivates us to investigate in more detail how toroidal rotation and rotational shear affect particle transport directly, when the underlying turbulence regime is not affected.

In this paper we keep the power input and mix the same while changing the injected torque using a combination of coinjected and counter-injected beams in predominantly ECH heated plasmas. This allows us to study the effect of rotation, rotational shear, and the $E \times B$ shear upon particle transport and confinement, while maintaining identical temperature profiles. As a result, the frequency of the dominant unstable mode is similar for all three discharges. Thus any changes in the density profile or the rotation profile will not be the result of a change in turbulence regime, but a direct result of a difference in injected torque and resulting $E \times B$ shear. As such, these results are different from those in AUG, where the changes in $u^{\prime}$ and $R / L_{n}$ are the result of a change in frequency of the most unstable mode. We find in DIII-D that $R / L_{n}$ at mid-radius does not strongly correlate with $u^{\prime}$. While particle confinement is not just determined by the local gradients at mid-radius,

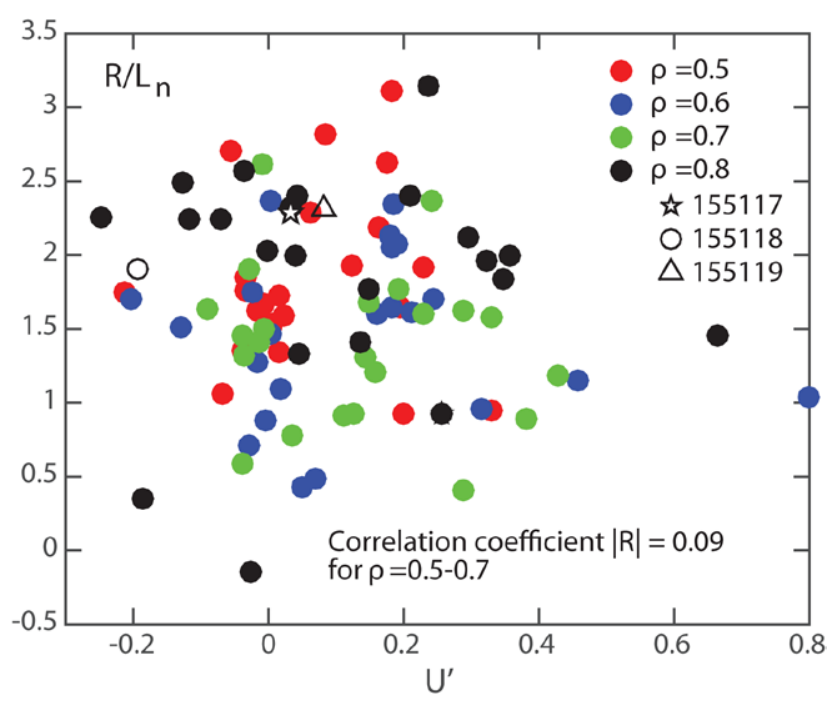

Figure 1. Normalized density gradient, $R / L_{n}$ as a function of toroidal rotation shear $u^{\prime}=-\frac{R}{v_{\mathrm{i}, \mathrm{th}}} \frac{\partial v_{\text {tor }}}{\partial R}$ for low density H-mode discharges in which the ratio of $T_{\mathrm{e}} / T_{\mathrm{i}}$ is varied.

we observe that the discharge with balanced injected torque has the lowest confinement as well as a the smallest $R / L_{n}$. A comparison of the calculated quasi-linear particle flux using TGLF with the experimental particle flux shows agreement on a qualitative (not quantitative) level with the co-injected discharge. However, for the balanced and the counter injected discharges, there is not even qualitative agreement. Neither does the linear growth rate as calculated with TGLF correlate with the observed density fluctuations. This indicates that more research needs to be done to validate reduced transport models in conditions without strong co-torque injection.

First we will discuss the experimental setup of these experiments in section 2 along with the linear stability calculations. In section 3 we will compare the experimental particle flux with quasi-linear TGLF calculations along with the experimentally measured perturbed transport coefficients. Next, in section 4 we will discuss the changes in turbulence characteristics with a focus on the changes in $E \times B$ shear along with the changes in growth rates and density fluctuations. We will conclude with a discussion and short summary.

\section{Experiment setup}

These experiments were performed in the DIII-D tokamak with major radius, $R \sim 1.67 \mathrm{~m}$ and minor radius, $a \sim 0.67 \mathrm{~m}$. The heating power is $\sim 5 \mathrm{MW}$ in these $\mathrm{H}$-mode plasmas, with a line averaged density of $3-4 \times 10^{19} \mathrm{~m}^{-3}$, see figure 2 . The toroidal magnetic field is $B_{\mathrm{T}}=1.9 \mathrm{~T}$, the plasma current is $I_{\mathrm{p}}=1.1 \mathrm{MA}$, which results in a $q_{95} \sim 4.2$ and a normalized plasma pressure, $\beta_{N}=1-1.5$, which allows us to avoid the locked mode limit while operating at low rotation and low density in DIII-D. In figure 2, at $2000 \mathrm{~ms}$, the initial 3.5 MW NBI power is reduced to $2 \mathrm{MW}$ and $3 \mathrm{MW}$ of $\mathrm{ECH}$ power is added. This is also when the torque is altered from purely coinjected to have an early L- to H-mode transition to maintain a low density in $\mathrm{H}$-mode. The torque is varied from $1.1 \mathrm{Nm}$ in 


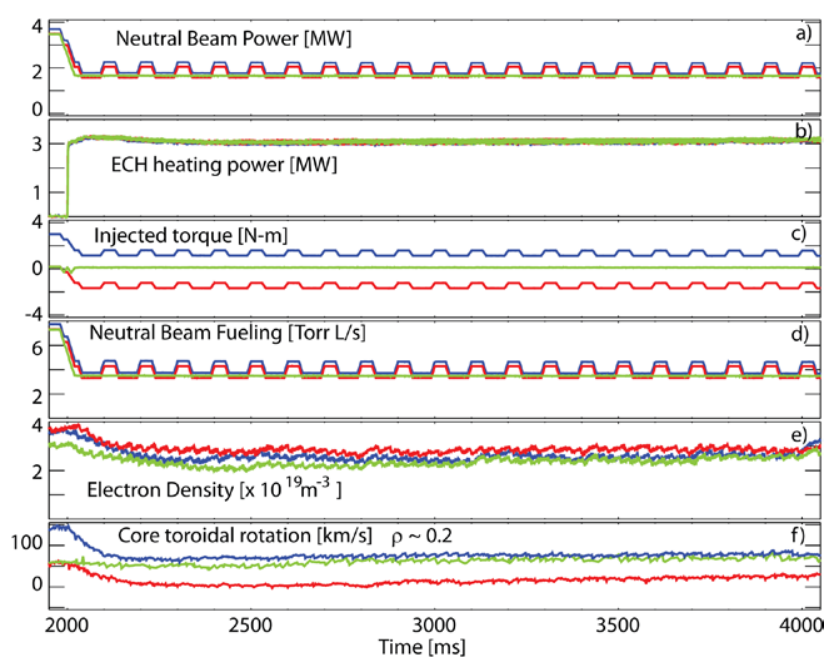

Figure 2. Time evolution of three DIII-D H-mode discharges with different torque injection. (a) The NBI heating power for each discharge and the (b) ECH injected power are the same. (c) The NBI torque injection is varied from co- to counter-injected. (d) The fueling from the NBI is the same for all three discharges. (e) The change in torque injection results in different line averaged electron density. (f) The core carbon rotation close to $\rho=0.3$ varies from corotating to zero rotation.

one discharge to $0 \mathrm{Nm}$ in the second discharge and $-1.6 \mathrm{Nm}$ in the third. The only fueling comes from the NBI and the plasma-wall interactions, there is no gas puff, nor pellet injections during the $\mathrm{H}$-mode phase of these discharges.

The change in injected torque results in a change in the core carbon rotation as measured by the Charge Exchange Recombination (CER) system [20]. Both the co-injected and the balanced injected discharge rotate in the co-current direction, while the counter injected discharge operates close to zero rotation outside $\rho \sim 0.3$ and rotates slightly in the counter direction inside $\rho \sim 0.3$, see figure 3. These results indicate that an intrinsic torque is present and is in all three cases in the co-current direction, similar as in Solomon et al [21]. The electron density is measured with the reflectometer system [22]. The changes in rotation affect particle confinement as well as the inverse density scale-length. The co- and counter-injected discharge have a similar $R / L_{n}$, whereas the balanced torque injected discharge has a much lower inverse density scale length as well as an overall lower electron density. Comparing $R / L_{n}$ with $u^{\prime}$ in figure 3 shows that there is no direct correlation between the local changes in $u^{\prime}$ and the local changes in $R / L_{n}$. This lack of correlation for these three discharges is emphasized in figure 1, where they are represented with an open symbol. Electron temperature profiles are measured using both the electron cyclotron emission (ECE) [23] and Thomson Scattering system [24]. The electron temperature profiles are well matched and only marginally affected by the changes in toroidal rotation. The ion temperature profile (measured with the CER system) is slightly higher and more peaked for the co-injected torque discharge.

Since the frequency of the most dominant unstable mode correlates with the change in $R / L_{n}$, we want to verify that a change in the mode frequency is not the driver for a change in the inverse density scale length $[2,11]$. We use TGLF [3,
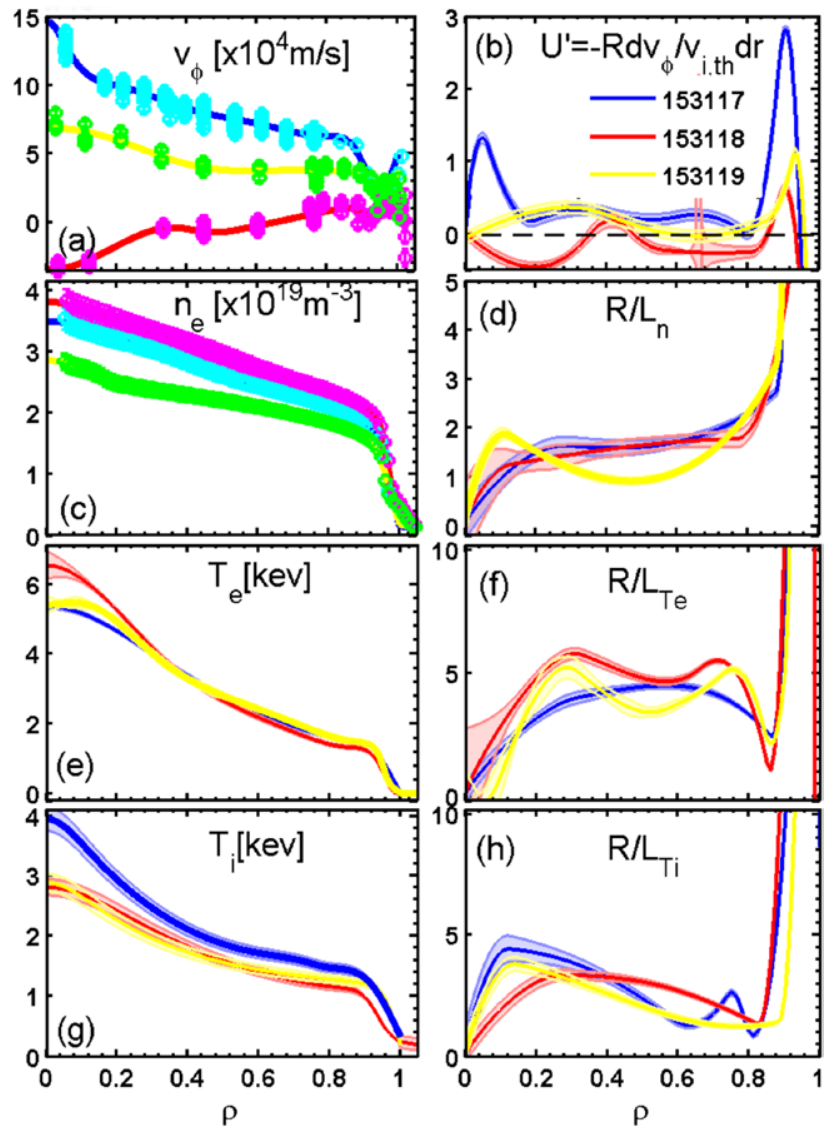

Figure 3. Experimental fitted profiles and their normalized gradients for all three discharges (dark blue and light blue $=$ co, yellow and green $=$ balanced and red and pink $=$ counter injected NBI). ((a), (b)) Due to the different injected torque the toroidal carbon rotation and its normalized gradient, $u^{\prime}$ is different for all three discharges. ((c), (d)) The electron density is the highest for the counter injected discharge and the lowest for the balanced injection. $R / L_{n}$ for the co- and counter injected discharge are fairly similar. ((e), (f)) The electron temperature profile is similar for all three discharges outside $\rho \sim 0.2$. ((g), (h)) The ion temperature is slightly higher for the co-injected discharge in comparison with the two other discharges.

25], a quasi-linear gyrokinetic code to calculate the linear growth rates and frequencies of the most unstable mode. TGLF has been benchmarked against GYRO [26] and is computational less expensive and agrees well with GYRO results when $k_{\theta} \rho_{s}<2$. Here $k_{\theta}$ is the poloidal wave-number, $\rho_{s}$ is the ion sound radius. Typically, the instabilities at small wave numbers dominate the changes in transport, although GYRO simulations have shown that non-linear coupling can have a substantial effect on the heat flux [27]. The experimental profile fits from figure 3 are used as input to TGLF to calculate the frequency of the most unstable mode (i.e. the mode with the largest growth rate) for $0<k_{\theta} \rho_{s}<1$, see figure 4. From $\rho=0.4-0.7$, the frequency of the most unstable mode is positive in the plasma frame, which is in the electron diamagnetic direction. As a result we can identify this mode as a Trapped Electron Mode (TEM), which is driven by both the electron density gradient and the electron temperature gradient. Inside $\rho=0.4$, the counter torque injected discharge remains in the TEM regime, whereas in the two other discharges the 


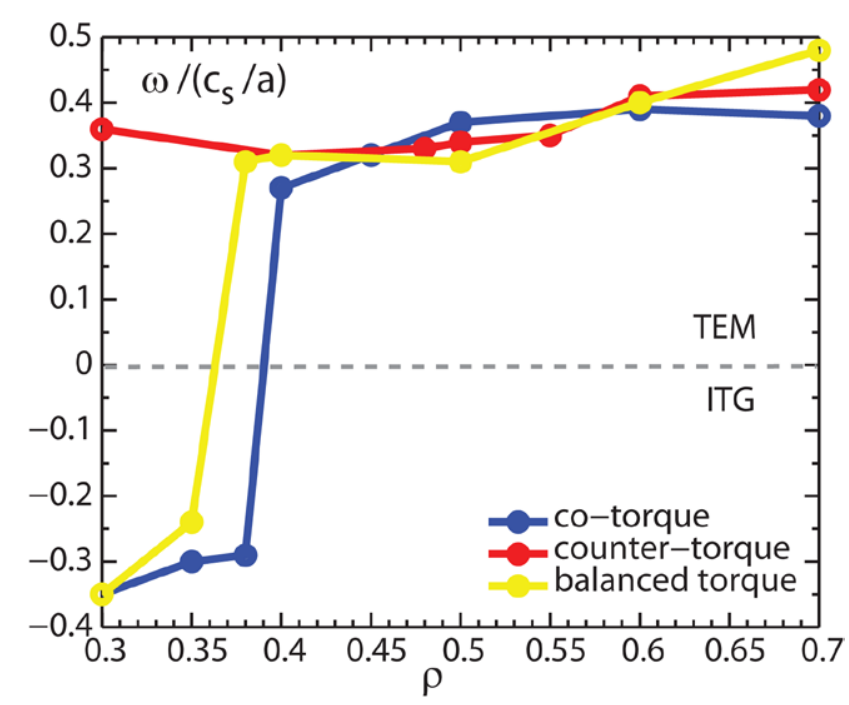

Figure 4. Calculation of the frequency of the most unstable mode in the plasma frame for $0<k_{\theta} \rho_{s}<1$ using TGLF. All three discharges are in TEM regime outside $\rho=0.4(\omega>0)$. Inside $\rho=0.4$, the counter torque injected plasma is still in the TEM regime, whereas the other two discharges switch to the ITG regime.

frequency switches to the ion-direction in the plasma frame. As a result, the dominant mode is now for these two discharges driven by Ion Temperature Gradient (ITG). While the dominant mode is not the only unstable mode, previous work has shown that the frequency of this mode plays an important role in determining the local density gradient $[2,11]$. In figure 4, we can observe that not only is the frequency of the most unstable mode in the same direction (for $\rho>0.4$ ), the magnitude of the frequency is also similar. We can therefore assume that the changes in $R / L_{n}$ are not the result of a change in mode frequency and focus on the role of the toroidal rotation in determining particle transport.

\section{Particle transport}

Using the particle balance equation $\frac{\partial n}{\partial t}=-\nabla \Gamma+S$, where $n$ is the electron density, $\Gamma$ is the particle flux and $S$ is the fueling source, we can determine the particle flux. In this case, we use ONETWO [28], a 1.5D transport code, to infer the fueling source from the beams and calculate the particle flux. Figure 5(a) shows the total particle flux from ONETWO. ONETWO uses a proxy to determine the particle source at the plasma edge based on the confinement time, which leads to an overestimation of the particle flux at the plasma edge. The NBI heating power is similar for all three discharges, and with NBI fueling directly proportional to the injected power, this results in a similar particle flux in the core. Figure 5(b) shows how steady-state transport varies for the three discharges, as represented by an effective diffusion coefficient, $D_{\text {eff }}=-\Gamma /(\partial n / \partial r)$. Please note, that this is an effective diffusion coefficient and thus does encompasses the particle pinch as well as the actual diffusion coefficient. While the particle flux is similar for all three discharges, the changes in local density gradients (see figure 3 ) result in the balanced injected discharge having a much larger effective outward transport than the co- and counter-torque injected discharges.

In figure 5(a) we also calculate the quasi-linear particle flux with TGLF in order to compare it to the experimental results. TGLF calculates the turbulent particle flux at each $k_{\theta} \rho_{S}$ for the different radii. By integrating over $k_{\theta} \rho_{s}=0-1$ we can extract the quasi-linear particle flux at different radial locations. We find that the calculated quasi-linear flux does not match the experimentally measured flux. In order to estimate how much the quasi-linear flux changes as a result of errors in the experimental measurements, we varied all the gradients by $10 \%$. These variations in gradients then determine the error-bars of the quasi-linear simulations. In the co-torque injected discharge, the quasi-linear flux is a factor 3 too small, but follows the same trends as the experimental flux. The counter-torque injected discharge has a negative quasi-linear flux between $\rho=0.5$ and $\rho=0.6$ and at larger radii the flux increase above experimental values. The quasi-linear particle flux calculations for the balanced-torque injected discharge also exceed the experimental observations outside $\rho=0.6$. Whereas, the calculation of the experimental flux is based on the radial integral of the source, the TGLF calculations are based upon local gradients.

As shown in figure 5, while the particle flux can be similar, particle transport can be very different. While we represented the changes in transport as an effective diffusion coefficient in figure 5 , in reality particle transport consists of a pinch, $v$, as well as diffusion component, $D: \Gamma=-D \frac{\partial n}{\partial r}+v n$. If this was a source-less plasma, we could assume that $1 / L_{n}=v / D$. This still does not allow us to extract the separate contributions of $D$ and $v$. So, in order to measure the pinch and diffusion contributions separately, we have to rely on the use of a perturbative technique. Since the 1980s and again in more recent years (due to improved temporal and spatial diagnostic capabilities), a modulated gas puff technique has been employed to measure the perturbed pinch, $v_{\mathrm{p}}$ and the perturbed diffusion coefficient, $D_{\mathrm{p}}[15,29-35]$. Adding a periodic small gas puff modulates the density profile with a fixed periodicity. We can extract both the amplitude and the phase of this modulation using Fourier analysis. Using the perturbed continuity equation as shown by Takenaga et al [36], the perturbed $D_{\mathrm{p}}$ and $v_{\mathrm{p}}$ can then be expressed in terms of the measured phase and amplitude. We refer the reader to previous work by Doyle et al. [15] and Mordijck et al [29, 30] with more details on this technique for DIII-D plasmas. Figure 6 shows the measured $D_{\mathrm{p}}$ and $v_{\mathrm{p}}$ of the three discharges. While the co-torque discharge and balanced torque discharge have similar transport coefficients, the counter-torque discharge has a stronger convective inward pinch. This matches well with the observation in figure 3(c) that the counter-torque discharge has the highest electron density. Caution needs to be applied into over-interpreting these results. The Fourier analysis only results in the extraction of the perturbed transport coefficients and does not include the steady-state and perturbed fueling sources. Ongoing work on JET includes a non-linear optimization to extract the steadystate transport coefficients, not just the perturbed transport coefficients [35]. However, this technique is still under development and cannot yet be applied to DIII-D plasmas. 

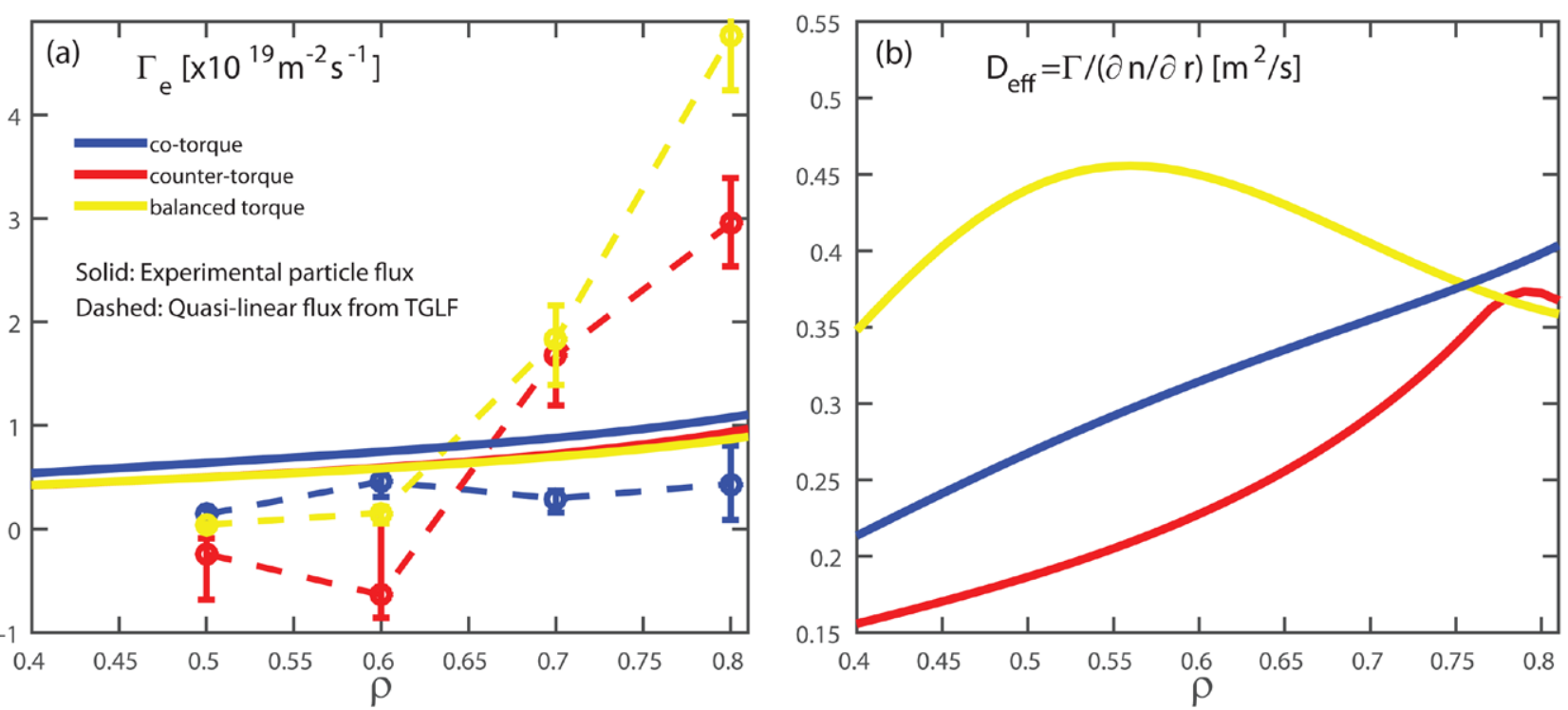

Figure 5. (a) The total electron particle flux (solid lines) is calculated using ONETWO, a 1.5D transport code; the dashed lines are the calculated quasi-linear electron flux from TGLF. The error bars on the quasi-linear flux calculations is based on a $10 \%$ variation of the fitted gradients. (b) The change in transport can be represented by an effective diffusion coefficient, $D_{\text {eff }}=\Gamma /(\partial n / \partial r)$.
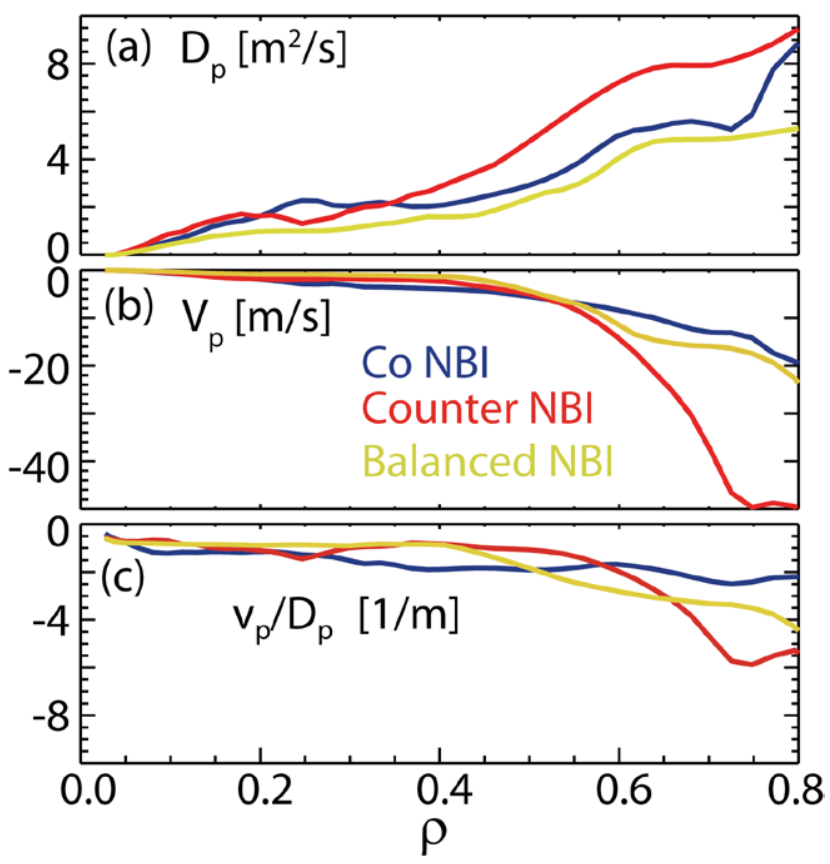

Figure 6. Perturbative transport measurements for the three discharges show that (a) the diffusive coefficients are similar in magnitude and (b) the convective coefficients show that countertorque discharge has a much larger inward pinch than the other two discharges outside $\rho=0.6$. (c) Shows the ratio of $v_{\mathrm{p}} / D_{\mathrm{p}}$.

\section{Turbulence and $E \times B$ shear}

Earlier we showed the there is no strong correlation between $u^{\prime}$ and $R / L_{n}$ in a database of DIII-D plasmas (see figure 1), nor is there a correlation between $u^{\prime}$ and $R / L_{n}$ in the set of three dedicated experiments in which the torque injection was varied (to vary the toroidal rotation), while keeping other parameters that affect turbulence the same. While $u^{\prime}$ acts as an off-diagnonal term in determining particle transport, its influence is small for main ions and electrons. However, toroidal rotation can also indirectly affect particle transport, through its contribution to the radial electric field $E_{\mathrm{r}}$ and thus the $E \times B$ shearing rate $[5,37]$ :

$$
\begin{gathered}
q E_{\mathrm{r}}=q\left(Z_{i} e n_{i}\right)^{-1} \nabla P_{i}-q v_{\theta_{i}} B_{\phi}+q v_{\phi i} B_{\theta} \\
\omega_{E \times B}=\frac{\left(R B_{\theta}\right)^{2}}{B}\left(\frac{\partial}{\partial \Psi}\right) \frac{E_{\mathrm{r}}}{R B_{\theta}}
\end{gathered}
$$

Here, $Z_{i}$ is the net particle charge of species $i, P_{i}$ is plasma pressure, $v_{i}$ is the plasma velocity and $\theta$ and $\phi$ denote poloidal and toroidal direction respectively, $B$ is the magnetic field and $\Psi$ is the poloidal flux. Figure 7 shows for the same database as figure 1 using the same profiles that there is a weak correlation for $\rho=0.5-0.7$ between the local $E \times B$ shearing rate and the local peaking of the density. Here the $E \times B$ shearing rate is based on measured quantities only (Carbon was the ion species used by the CER system), which have been fitted (see figure 1). While the density gradient has a contribution in the $\omega_{E \times B}$, there were large variations in the electron and ion temperature gradients as well as rotation to eliminate any trends related to the density gradient. In the next section we will study the role of the $E \times B$ shearing rate in more detail for the discharges in which we varied the torque injection.

\subsection{Growth rate versus $E \times B$ shearing rate}

Gradients drive micro-instabilities in tokamak plasmas, which results in the creation of eddies that drive turbulent transport. These eddies can be torn apart and/or even suppressed by a sheared flow [5, 6, 38-40]. Sheared flow, such as the $E \times B$ shear, affects turbulence in two ways [5]; through nonlinear de-correlation, which includes the reduction in the turbulence radial correlation length, phase, and fluctuation size; or through linear stabilization, which enhances the damping of 


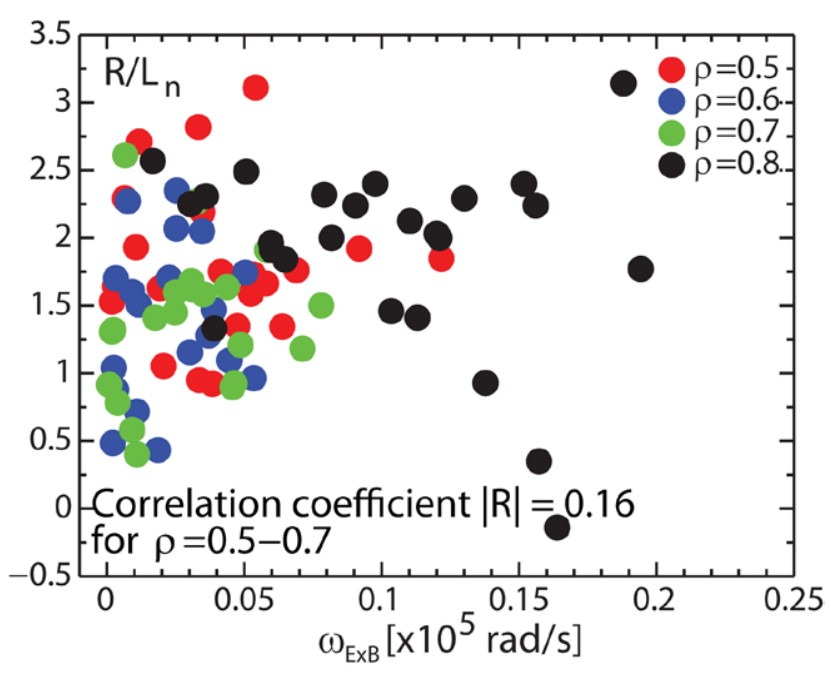

Figure 7. This figure shows the normalized density gradient as a function of the $E \times B$ shearing rate for the same database of $\mathrm{H}$-mode discharges as figure 1 . The different colors represent different radial locations.

turbulence by coupling the unstable modes to nearby, stable modes, thus improving the overall stability of the system. In the pedestal region, the radial electric field (and thus the $\omega_{E \times B}$ rate) is dominated by the pressure gradient, deeper inside the core, the toroidal rotation is more important [41]. In our set of three discharges, due to the changes in input torque, we change the toroidal rotation and the $E \times B$ shear, see figure 8 . From $\rho=0.6-0.8$ the change in toroidal rotation has the strongest effect. Outside $\rho=0.8$ the changes in the pressure gradient dominate and inside $\rho=0.6$ the shearing rate is small and the local difference between the discharges are related to fitting choices. The discharge with balanced torque injection has the lowest $\omega_{E \times B}$, while the co- and counter torque injected discharges have a higher shearing rate from $\rho=0.6-0.8$.

The shearing rate by itself can be an indication of whether turbulent transport will be suppressed, but a better indication is to compare the linear growth rate of the instabilities with the $E \times B$ shearing rate. In a simplified picture, without including non-linear effects, the rule of thumb is that if the linear growth rate is smaller than the shearing rate, the mode will be suppressed, whereas if the linear growth rate is larger than the shearing rate, the mode will not be suppressed by the shearing rate. Figure 9 shows the maximum linear growth rates for $k_{\theta} \rho_{s}=0.1-0.5$ along with $\omega_{E \times B}$. For the co- (figure 9 (a)) and the counter-torque injected cases (figure 9(b)) $\omega_{E \times B}$ is larger than or of similar magnitude as the maximum linear growth rate. Whereas for the balanced torque injected discharge, the average linear growth rate is significantly larger than the $E \times B$ shearing rate from $\rho=0.6-0.85$. This suggests that the balanced injected discharge will not be able to suppress turbulent transport outside the mid-radius up to the top of the pedestal, based on the simplified picture. However, this is in a simplified linear picture, as mentioned earlier, the $E \times B$ shearing rate can also result in the non-linear de-correlation of the turbulent eddies. Whether the modes at smaller $k_{\theta} \rho_{s}$ are suppressed or whether it is the de-correlation of these modes that results in a decrease in transport and improvement in

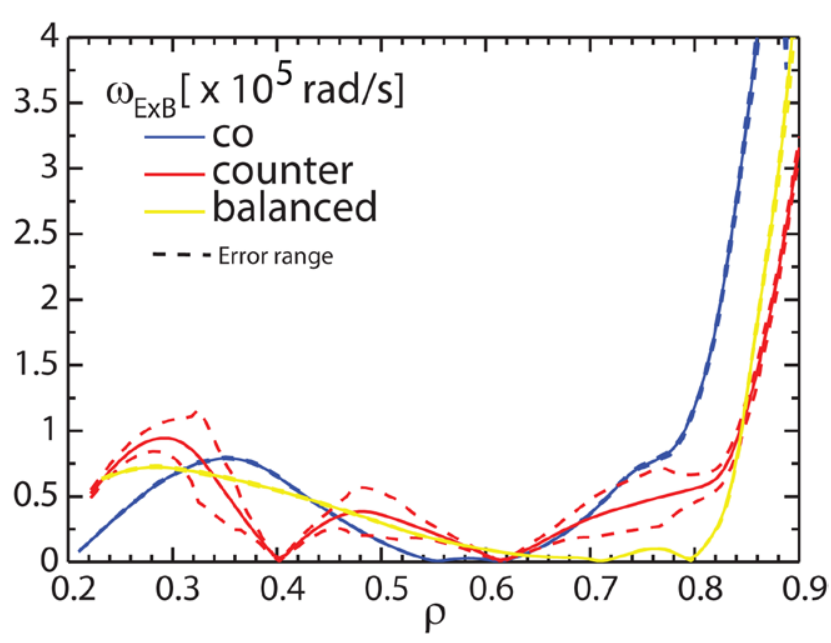

Figure 8. The $E \times B$ shearing rate (using Hahm-Burrell [5]) is highest for the co-injected discharge (blue) from $\rho \sim 0.6-0.9$. In the same radial region, the $E \times B$ shear is smallest for the balanced torque injected discharge (yellow).

confinement, is impossible to tell without 2D experimental visualization of the actual eddies. Also, these linear simulations do not address the role of smaller scale turbulence and the full non-linear coupling between the different modes. This rule of thumb on the role of the $E \times B$ shear has been observed before on DIII-D, when the toroidal rotation is strongly modified using Resonant Magnetic Perturbations [29].

\subsection{Density fluctuations}

To investigate the changes in turbulence we compare the density fluctuations measured by the Doppler BackScattering [42], see figure 10. The DBS measures density fluctuations at the intermediate scale, in this case the range in $k_{\theta} \rho_{s}=1.6-2.8$. This intermediate scale is typically associated with the TEM unstable regime. We observe in figure 10(a) that density fluctuations are similar outside $\rho=0.9$. From $\rho \sim 0.6-0.75, \tilde{n} / n$ is slightly higher for the balanced torque discharge. From $\rho \sim 0.75-0.9$, there is no data for the co and balanced discharge, but at $\rho=0.9$, data seems to suggest that the density fluctuations can vary rapidly over a small radial extend and that the counter injected discharge has a much higher $\tilde{n} / n$, than the other two discharges. From $\rho \sim 0.7-0.9$ is also the radial location where a strong increase in the perturbed particle pinch measurement is observed for the counter-torque injected discharge, see figure 6.

Figure 10(b) shows the average linear growth rate for the same $k_{\theta} \rho_{s}$ scale. In a simplified linear picture, the growth rates should be equivalent to the actual density fluctuations at the same scale. Accounting for the fact that there is no data for the co and balanced injected discharge, it is impossible to compare the trends between $\rho=0.7-0.9$. Outside this area, the linear growth rates seem to be in agreement qualitatively with the observed density fluctuations. The values for the balanced torque injected discharge show no indication to increase between $\rho=0.7-0.9$ as shown in the simulations, but neither an increase nor decrease in the density fluctuation amplitude can be confirmed. 


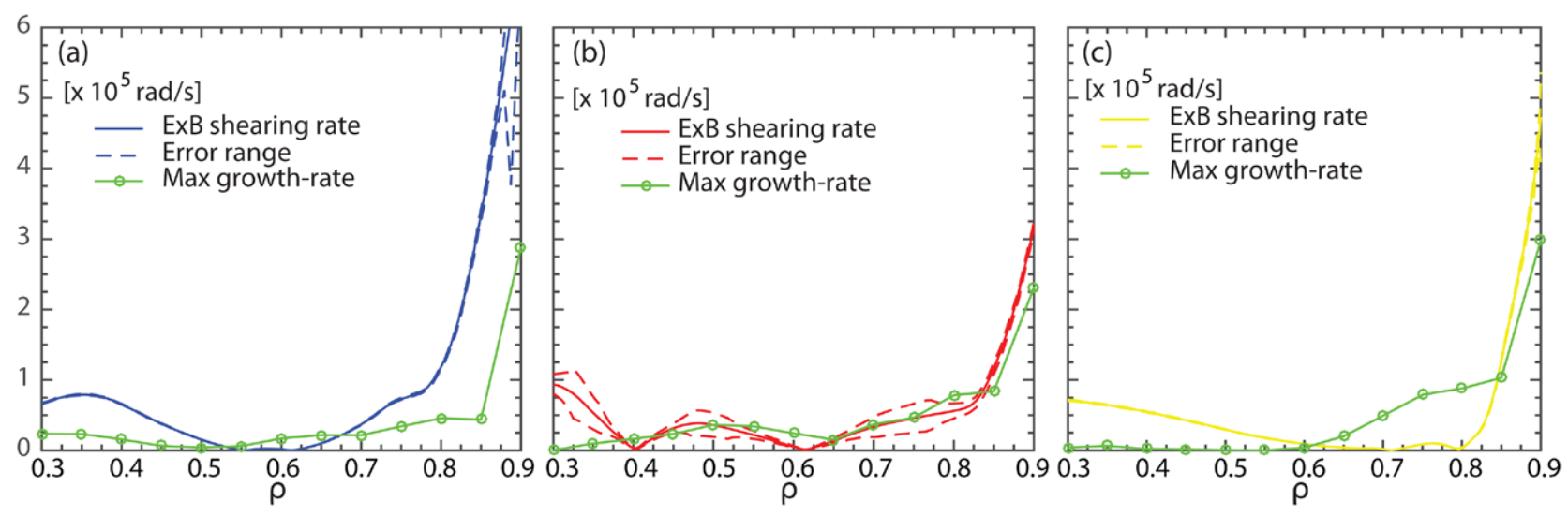

Figure 9. We compare the $E \times B$ shearing rates to the maximum growth rates for $k_{\theta} \rho_{s}=0.1-0.5$. The $E \times B$ shearing rates are for (a) the cotorque is larger than the linear growth rate from $\rho \sim 0.7-0.9$. (b) In the counter-torque (Red) discharge the $E \times B$ shear and the linear growth rate have the same magnitude. (c) For the balanced torque discharge (Yellow) the linear growth rate is larger than the $E \times B$ shearing rate.
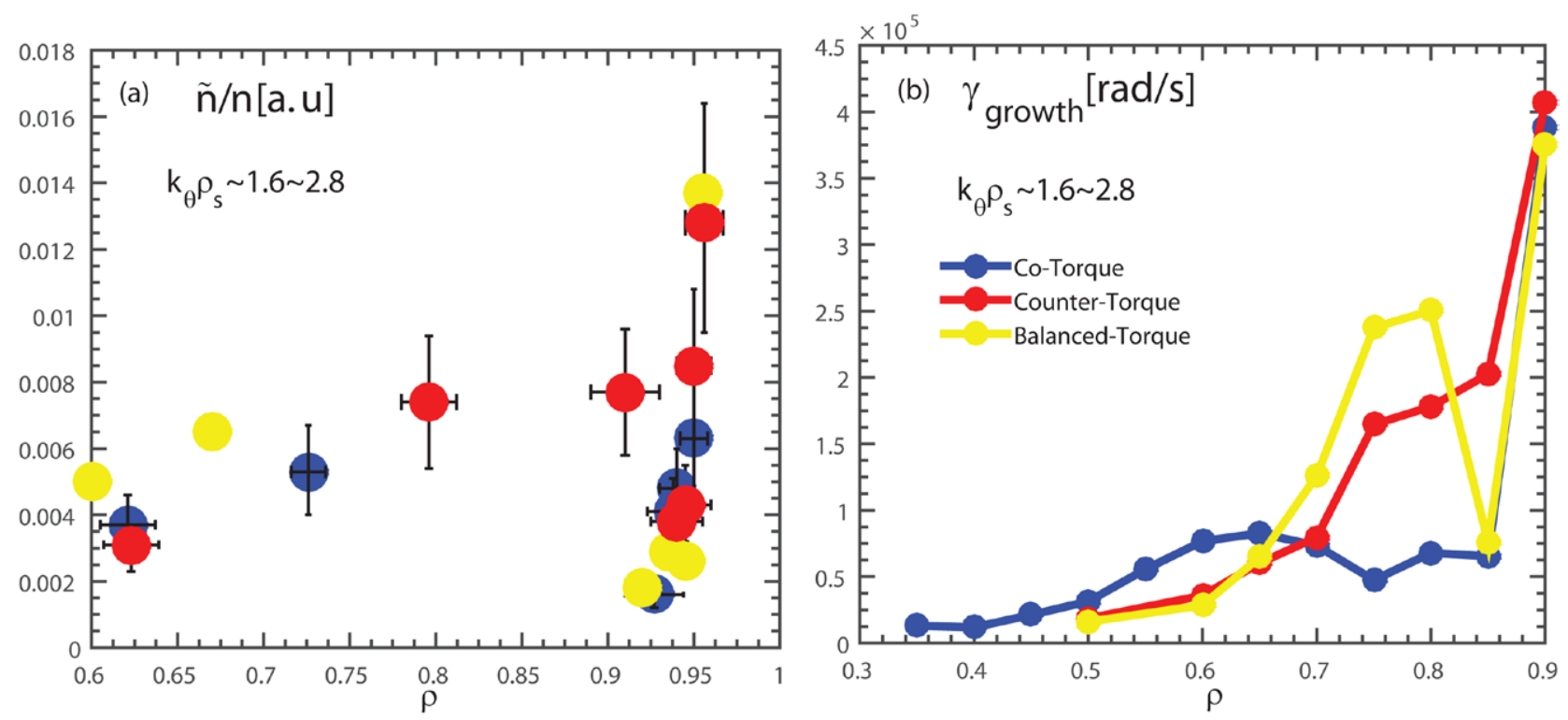

Figure 10. (a) We measure the intermediate scale density fluctuations $\left(k_{\theta} \rho_{s}=1.6-2.8\right)$ using the DBS for the three discharges. (b) We compare the density fluctuations to the average linear growth rate for the same $k_{\theta} \rho_{s}=1.6-2.8$.

\section{Discussion}

In this paper we seek to study the effects of rotation upon particle transport. On AUG, when the frequency of the dominant unstable mode is varied by changing the $T_{\mathrm{e}} / T_{\mathrm{i}}$ ratio, a correlation between $R / L_{n}$ and $u^{\prime}$ is observed [11]. In this paper, we do not observe a strong correlation between $R / L_{n}$ and $u^{\prime}$, see figure 1 . In a dedicated study to investigate the role of the toroidal rotation upon particle transport, we find that if the frequency of the dominant unstable mode remains similar and only the input torque is varied, there is no correlation between $u^{\prime}$ and $R / L_{n}$. This suggests that the correlation between $u^{\prime}$ and $R / L_{n}$ is the result of a change in the underlying turbulence regime and not a result of a change in off-diagonal particle transport.

The changes in toroidal rotation however feed into the $E \times B$ shearing rate and by varying the injected torque we were able to vary the $E \times B$ shear substantially from $\rho=0.6-0.8$. The ability of the $E \times B$ shear from mid-radius to the top of the pedestal to regulate particle confinement was also found previous in QH-mode [43] and during the application of Resonant Magnetic Perturbations (RMPs) [29]. In both previous results, when the linear growth rate for small $k_{\theta} \rho_{s}$ from mid-radius to the top of the pedestal is larger than the $E \times B$ shear, a reduction in particle confinement is observed. While in the QH-mode and the RMP H-mode experiments on DIII-D, the underlying linear stability is altered (due to changes in temperature gradients), in this dedicated set of three discharges the gradients are similar and, as a result, the frequency of the most unstable mode is similar for all three discharges in which the torque was varied. A similar effect was observed in the formation of an Internal Transport Barrier (ITB) on TFTR, where the magnetic shear reversal could not explain the improvement in confinement [44]. Counter to the experiments described before, the change in the $E \times B$ shear in this case is not the result of a change in toroidal rotation, but is due to the steep gradients formed in the ITB region. In this case, the suppression of turbulence is more similar to 
the formation of the pedestal during the L- to H-mode transition, where eventually the gradients in the pedestal provide the shear to maintain the pedestal through suppression of turbulent transport.

However as is shown in this paper, the linear and quasilinear gyrokinetic simulations show some disagreement with experimental observations on microscopic and macroscopic scales. There is no correlation between linear growth rates and experimental density fluctuations nor does quasi-linear particle flux match the experimental particle flux. One explanation could be that non-linear effects play an important role and that full non-linear gyrokinetic simulations would result in better agreement. For example, non-linear multi-scale gyrokinetic simulations have already shown to increase the heat flux by $30 \%$ in comparison with ITG-only non-linear gyrokinetic simulations [45]. As of yet, it is unclear whether fully non-linear multi-scale gyrokinetic simulations would result in an increase of the particle flux by a factor 3 or result in a reversal in the particle flux from inward to outward or a decrease in particle flux at other radii. Future work will include full non-linear gyrokinetic simulations to assess how much they diverge from the quasi-linear simulations with respect to particle transport.

The perturbed transport coefficients indicate that the changes in transport are not just the result of changes in outward diffusion, but also inward convection. However, these are perturbed transport coefficients and it is unclear whether they accurately reflect the steady-state transport coefficients. Although the perturbations to the density are small, this can still affect the local gradients. In order to connect the perturbed transport coefficients to the background steady state transport coefficients, a non-linear optimization is needed, which includes not only the perturbed density profiles, but also the steady-state density profile, the steady-state and perturbed fueling sources. Initial progress on this topic has been made on JET, but the technique is still being tested and developed [35]. Although perturbed techniques to measure particle transport coefficients have been around since the $80 \mathrm{~s}$, more work needs to be done to connect them to steady-state transport coefficients. One interesting observation is that we measure in the counter-torque plasma a strong inward particle pinch, which occurs at the same radii as the strong increase in intermediate scale density fluctuations. A similar correlation between a strong increase in perturbed inward particle pinch and an increase in intermediate scale density fluctuations is also observed in plasmas in which the $T_{\mathrm{e}} / T_{\mathrm{i}}$ is varied [30].

\section{Summary}

In this paper we present the first dedicated study on how changes in toroidal rotation affect particle transport and confinement, without changing the underlying turbulence drive. We vary the toroidal rotation by changing the injected torque and we find that there is a correlation between $R / L_{n}$ and the $E \times B$ shear. When the $E \times B$ shear is lower than the linear growth rate at small $k_{\theta} \rho_{s}$ outside mid-radius, this results in a decrease in particle confinement. As a result, the discharge without external net torque results in the lowest particle confinement of the three discharges. Considering that ITER will not be able to rely on externally injected torque, this might negatively affect particle confinement on ITER. Perturbed transport coefficients indicate that changes in transport are not just related to an increase in outward diffusion. In the counter-torque injected discharge, which has the best particle confinement a strong increase in the perturbed inward pinch is observed at the plasma edge and intermediate scale density fluctuation at the same radius. Finally, we observe that quasilinear gyrokinetic simulations of the particle flux using TGLF do not match experimental observations. This suggests that in order to improve confidence in predicting density profiles, advances need to be made in quasi-linear gyrokinetic simulations for low torque plasmas.

\section{Acknowledgment}

This material is based upon work supported by the US Department of Energy, Office of Science, Office of Fusion Energy Sciences, using the DIII-D National Fusion Facility, a DOE Office of Science user facility, under Awards DE-SC0007880, DE-FC02-04ER54698, and DE-FG0208ER54984. DIII-D data shown in this paper can be obtained in digital format by following the links at https://fusion.gat. com/global/D3D_DMP.

\section{References}

[1] Luxon J L 2002 A design retrospective of the DIII-D tokamak Nucl. Fusion $\mathbf{4 2} 614$

[2] Angioni C et al 2011 Intrinsic toroidal rotation, density peaking, and turbulence regimes in the core of tokamak plasmas Phys. Rev. Lett. 107215003

[3] Staebler G M, Kinsey J E and Waltz R E 2005 Gyro-Landau fluid equations for trapped and passing particles Phys. Plasmas 12102508

[4] Fitzpatrick R and Hender T C 1991 The interaction of resonant magnetic perturbations with rotating plasmas Phys. Fluids B: Plasma Phys. 3 644-73

[5] Burrell K H 1997 Effects of ExB velocity shear and magnetic shear on turbulence and transport in magnetic confinement devices Phys. Plasmas 41499

[6] Biglari H, Diamond P H and Terry P W 1990 Influence of sheared poloidal rotation on edge turbulence Phys. Fluids B 2 1-4

[7] Aiba N, Shiraishi J and Hirota M 2013 Impact of plasma rotation on the linear physics of resistive wall modes in tokamaks Plasma Phys. Control. Fusion 55074002

[8] Chapman I T et al, the MAST, TEXTOR Teams and JET EFDA Contributors 2007 The physics of sawtooth stabilization Plasma Phys. Control. Fusion 49 B385

[9] Garzotti L et al 2012 Simulations of density profiles, pellet fuelling and density control in ITER Nucl. Fusion 52013002

[10] Lawson J 1957 Some criteria for a power producing thermonuclear reactor Proc. Phys. Soc. B 70 6-10

[11] Angioni C, Camenen Y, Casson F J, Fable E,

McDermott R M, Peeters A G and Rice J E 2012 Offdiagonal particle and toroidal momentum transport: a survey of experimental, theoretical and modelling aspects Nucl. Fusion 52114003 
[12] Angioni $\mathrm{C}$ et al and Contributors to the EFDA-JET. Workprogramme 2007 Scaling of density peaking in $\mathrm{H}$-mode plasmas based on a combined database of AUG and JET observations Nucl. Fusion 471326

[13] Angioni C, Candy J, Fable E, Maslov M, Peeters A G, Waltz R E and Weisen H 2009 Particle pinch and collisionality in gyrokinetic simulations of tokamak plasma turbulence Phys. Plasmas 16060702

[14] Greenwald M, Angioni C, Hughes J W, Terry J and Weisen H 2007 Density profile peaking in low collisionality H-modes: comparison of alcator C-Mod data to ASDEX Upgrade/JET scalings Nucl. Fusion $\mathbf{4 7}$ L26

[15] Doyle E J et al 2012 Particle transport results from collisionality scans and perturbative experiments on DIII-D In Conf. Proc. of 24th IAEA Fusion Energy Conf. (San Diego, USA, 2012)

[16] Weisen H, Zabolotsky A, Maslov M, Beurskens M, Giroud C, Mazon D and JET-EFDA Contributors 2006 Scaling of density peaking in Jet H-modes and implications for ITER Plasma Phys. Control. Fusion 48 A457

[17] Tala T et al and JET Contributors 2015 Dimensionless collisionality scans for core particle transport in JET 42nd EPS Conf. on Plasma Physics

[18] Angioni C, Peeters A G, Garbet X, Manini A, Ryter F and ASDEX. Upgrade Team 2004 Density response to central electron heating: theoretical investigations and experimental observations in ASDEX Upgrade $\mathrm{Nucl}$. Fusion 44827

[19] Angioni C, McDermott R M, Fable E, Fischer R, Ptterich T, Ryter F, Tardini G and the ASDEX Upgrade Team 2011 Gyrokinetic modelling of electron and boron density profiles of H-mode plasmas in ASDEX Upgrade Nucl. Fusion $\mathbf{5 1} 023006$

[20] Grierson B A, Burrell K H, Solomon W M and Pablant N A 2010 Deuterium velocity and temperature measurement on the DIII-D tokamak Rev. Sci. Instrum. 81 10D735

[21] Solomon W M et al 2010 Mechanisms for generating toroidal rotation in tokamaks without external momentum input Phys. Plasmas 17056108

[22] Zeng L, Wang G, Doyle E J, Rhodes T L, Peebles W A and Peng Q 2006 Fast automated analysis of high-resolution reflectometer density profiles on DIII-D Nucl. Fusion 46 S677

[23] Truong D D and Austin M E 2014 High spatial resolution upgrade of the electron cyclotron emission radiometer for DIII-D tokamak Rev. Sci. Instrum. 85 11D814

[24] Ponce-Marquez D M, Bray B D, Deterly T M, Liu C and Eldon D 2010 Thomson scattering diagnostic upgrade on DIII-D Rev. Sci. Instrum. 81 10D525

[25] Kinsey J E, Staebler G M and Waltz R E 2008 The first transport code simulations using the trapped gyro-landaufluid model Phys. Plasmas 15055908

[26] Candy J and Waltz R E 2003 An eulerian gyrokinetic-maxwell solver J. Comput. Phys. 186 545-81

[27] Howard N T, White A E, Greenwald M, Holland C and Candy J 2014 Multi-scale gyrokinetic simulation of alcator C-Mod tokamak discharges Phys. Plasmas 21032308
[28] Pfeiffer W W et al 1980 ONETWO: a computer code for modeling plasma transport in tokamaks Technical Report GA-A16178 General Atomics Company Report

[29] Mordijck S et al 2012 Changes in particle transport as a result of resonant magnetic perturbations in diii-d Phys. Plasmas 19056503

[30] Mordijck S et al 2015 Particle transport in low-collisionality H-Mode plasma on DIII-D Nucl. Fusion 55113025

[31] O'Rourke J, Rimini F G and Start D F H 1992 Perturbative measurements of the electron transport matrix using ICRF power modulation Nucl. Fusion 321861

[32] Gentle K W, Cima G, Gasquet H, Hallock G A, Phillips P E, Rowan W L, Watts C, Gehre O and the ASDEX Upgrade Team 1995 Characteristics of equilibrium and perturbed transport coefficients in tokamaks Phys. Scr. 52411

[33] Lopes N J 1995 Perturbative transport studies in fusion plasmas Plasma Phys. Control. Fusion 37799

[34] Jacchia A, Mantica P, De Luca F and Gorini G 1991 Determination of diffusive and nondiffusive transport in modulation experiments in plasmas Phys. Fluids B 3 3033-40

[35] Salmi A et al and JET Contributors 2015 Particle source and edge transport studies in JET H-mode gas puff modulation experiments 41st EPS Conf.

[36] Takenaga H, Nagashima K, Sakasai A, Oikawa T and Fujita T 1998 Determine of particle transport coefficients in reversed shear plasma of JT-60U Plasma Phys. Control. Fusion 40183

[37] Hahm T S and Burrell K H 1995 Flow shear induced fluctuation suppression in finite aspect ratio shaped tokamak plasma Phys. Plasmas 2 1648-51

[38] Doyle E J et al 1993 Turbulence and transport reduction mechanisms in the edge and interior of DIII-D H-mode plasmas Proc. 14th Int. Conf. on Plasma Physics and Controlled Nuclear Fusion Research, Würzburg, (IAEA, Vienna, 1993) vol 1235

[39] Terry P W 2000 Suppression of turbulence and transport by shear flow Rev. Mod. Phys. 72109

[40] Waltz R E, Kerbel G D and Milovich J 1994 Toroidal gyrolandau fluid model turbulence simulations in a nonlinear ballooning mode representation with radial modes Phys. Plasmas 12229

[41] McDermott R M et al and Alcator C-Mod Team 2009 Edge radial electric field structure and its connections to h-mode confinement in Alcator C-Mod plasmasa Phys. Plasmas 16056103

[42] Hillesheim J C, Peebles W A, Rhodes T L, Schmitz L, Carter T A, Gourdain P A and Wang G 2009 A multichannel, frequency-modulated, tunable doppler backscattering and reflectometry system Rev. Sci. Instrum. 80083507

[43] Garofalo A M et al 2011 Advances towards QH-mode viability for ELM-stable operation in ITER Nucl. Fusion $\mathbf{5 1} 083018$

[44] Mazzucato E et al 1996 Turbulent fluctuations in TFTR configurations with reversed magnetic shear Phys. Rev. Lett. $773145-8$

[45] Howard N T, White A E, Reinke M L, Greenwald M, Holland C, Candy J and Walk J R 2013 Validation of the gyrokinetic model in ITG and TEM dominated L-mode plasmas Nucl. Fusion 53123011 